\title{
Vehicle parameter estimation using a model-based estimator
}

\author{
Matilde Paiano ${ }^{\mathrm{a}}$, Giulio Reina ${ }^{\mathrm{a}}$, Jose-Luis Blanco ${ }^{\mathrm{b}}$ \\ ${ }^{a}$ Dept. of Engineering for Innovation, University of Salento, Via Annesano, 73100 Lecce, \\ Italy \\ ${ }^{b}$ Dept. of Engineering, Universidad of Almería, 04120 Almería, Spain
}

\begin{abstract}
In the last few years, many closed-loop control systems have been introduced in the automotive field to increase the level of safety and driving automation. For the integration of such systems, it is critical to estimate motion states and parameters of the vehicle that are not exactly known or that change over time. This paper presents a model-based observer to assess online key motion and mass properties. It uses common onboard sensors, i.e. a gyroscope and an accelerometer and it aims to work during normal vehicle maneuvers, i.e. turning motion and passing. First, basic lateral dynamics of the vehicle is discussed. Then, a parameter estimation framework is presented based on a Extended Kalman filter. Results are included to demonstrate the effectiveness of the estimation approach and its potential benefit towards the implementation of adaptive driving assistance systems or to automatically adjust the parameters of onboard controllers.
\end{abstract}

Keywords:

Vehicle state estimation, Extended Kalman filter, mass estimation, adaptive estimation, vehicle lateral dynamics 


\section{Introduction}

2 The performance of driving assistance systems may be improved if the 3 unknown parameters of the underlying vehicle model can be measured and

4 updated. Weight of the vehicle, road adhesion, drag coefficient and tire cor-

5 nering stiffness are examples of unknown parameters. Specifically, the mass

6 of a vehicle plays an important role in terms of acelleration/braking, han-

7 dling and comfort performance. However, it is subject to variations during

s operating conditions. For heavy duty vehicles, the weight can vary as much - as $400 \%$ according to the payload. Anti-lock Braking System (ABS), Electronic Stability Program (ESP), and Adaptive Cruise Control (ACC) are all examples of controllers that relies on the accurate value of the vehicle mass for proper operation. Current implementations work with the assumption of 13 a maximum payload to provide passengers with the highest level of comfort 14 and safety independently of the load conditions. Therefore, the introduction 15 of automatic load detection systems will be the basis for some more vehicle improvements. As soon as onboard controllers can incorporate information about the actual vehicle weight in their response, this will enable them to provide even more efficient comfort and support for drivers. Therefore, they may achieve better results by taking the actual vehicle weight into account.

The paper is organized as follows. Section 2 surveys related research point21 ing out the novel contributions of the proposed approach. In Section 3, basic 22 concepts of lateral vehicle dynamics are recalled that serve as a basis for the 23 model-based observer described in Section 4. The proposed method recur24 sively updates the vehicle mass providing flexibility to the observer. The 25 technique is studied in a sequence of simulations, as detailed in Section 5, 
26 attesting to the feasibility of the proposed approach. Finally, Section ??

${ }_{27}$ concludes the paper.

\section{Related Work}

20 Vehicle parameter estimation is a critical issue connected with the inte-

30 gration of onboard control systems, especially when these parameters change

31 over time or they are difficult to measure directly [1]. Therefore, the availabil-

32 ity of an online estimation method for the vehicle's weight would be valuable

33 as it greatly affects its behaviour in terms of longitudinal, lateral and vertical

34 dynamics. In addition, as the level of driving automation increases, there are

35 more control modules that may benefit from on-line estimation of the vehi-

36 cle's load, including longitudinal control of platoons of vehicles [2], emission

${ }_{37}$ reduction and transmission control [3].

38 In general, the methods proposed in the literature can be classified in two

3o broad families: sensor-based and model-based methods. In sensor-based

40 methods, an additional dedicated sensor is employed. As an example, the

${ }_{41}$ vehicle's weight can be estimated by monitoring the suspension deflection us-

${ }_{42}$ ing strain gages [4] or an electro-magnetic sensor [5]. Recently, Continental

43 has announced a future generation of sensors, which will be fitted directly

44 underneath the tread of the tire to measure the total weight of the vehicle [6].

45 As the contact patch of the tire increases with the vertical load, by detecting

${ }_{46}$ the size of the contact area, it will be possible to infer information about the

${ }_{47}$ vehicle's weight.

48 In contrast, model-based (or indirect) methods use a model of the vehicle,

40 software algorithms and existing sensors (different from direct mass sensors) 
so to estimate the unknown parameter. They represent a promising solution in 51 terms of cost-effectiveness (no extra hardware). Most of the research in this 52 field focuses on the longitudinal dynamic problem. Examples of adaptive ${ }_{53}$ controllers for vehicle speed control can be found in [7], [8]. Simultaneous 54 estimation of vehicle mass and road grade has been studied by many. For ${ }_{55}$ example, an adaptive control scheme for longitudinal control of heavy-duty ${ }_{56}$ vehicles is proposed in [9], whereas [10] propose the use of Recursive Least 5) Squares (RLS) with multiple forgetting factors. An EKF approach is pro58 posed in [11] using two possible measurement configurations: the first one 50 using only the vehicle speed and a second one in conjunction with an addiso tional longitudinal accelerometer. The advantages of using an accelerometer 61 are also shown in [12] where a method to estimate vehicle mass and road 62 grade using an EKF is presented. An active estimator is proposed in [13] to 63 enhance parameter identifiability through the use of an EKF for parameter 64 estimation and model predictive control to control vehicle speed.

${ }_{65}$ A body of research also deals with vertical dynamics for vehicle mass esti66 mation. Often, these methods assume that the terrain profile is known or б estimated [14], [15], [16], [17]. In [18] the vertical response is analyzed in the 68 frequency domain to reveal important resonance frequencies related to the 6o value of the sprung mass.

70 Another strand of the research on mass estimation focuses on powertrain dy71 namics. For example, in [19] the mass of a truck is estimated by measuring 72 vehicle speed and engine torque and angular velocity during acceleration and 73 gear shifting stages, resulting in an accuracy of $10 \%$.

${ }_{74}$ In this work, an adaptive observer for automatic weight estimation is pre- 
sented based on the lateral dynamic model of the vehicle, which represents a

76 novel contribution to the literature. An EKF formulation is proposed where

$\pi$ the varying parameter is included in the state vector and continuously up-

${ }_{78}$ dated using current sensory data. This formulation has general value and it

79 may be used to track any other time-varying parameter provided that the

so observability condition is satisfied.

\section{3. Vehicle model}

The lateral behaviour is an important aspect in vehicle design, as it directly affects handling and comfort properties. Figure 1 shows the two degreeof-freedom model used in this research commonly known as the "bicycle" or "single track" model that holds under the following simplifications [20]: no weight transfer, constant vehicles longitudinal velocity $u$, equal internal and external dynamics so that tires of the same axle can be collapsed, linear range of the tires, rear-wheel drive, negligible motion resistance, and small angle approximation. The two degrees of freedom are the vehicle lateral velocity $v$ and yaw rate $r$. The equations of motion for the single-track model are given by:

$$
\begin{aligned}
& \dot{v}=-\left(\frac{C_{F}+C_{R}}{M u}\right) v-\left(\frac{C_{F} a-C_{R} b}{M u}+u\right) r+\frac{C_{F} \delta}{M} \\
& \dot{r}=-\left(\frac{C_{F} a+C_{R} b}{I u}\right) v-\left(\frac{C_{F} a^{2}+C_{R} b^{2}}{I u}\right) r+\frac{C_{F a \delta}}{I}
\end{aligned}
$$

where $M$ and $I$ are the mass and the rotational inertia of the vehicle, $\delta$ is the front steer angle, $a$ and $b$ are the distance of the centre of gravity $G$ from the front and rear axle, respectively, and $C_{F}$ and $C_{R}$ are the front and rear tire cornering stiffness. In the study of lateral dynamics it is often useful to 


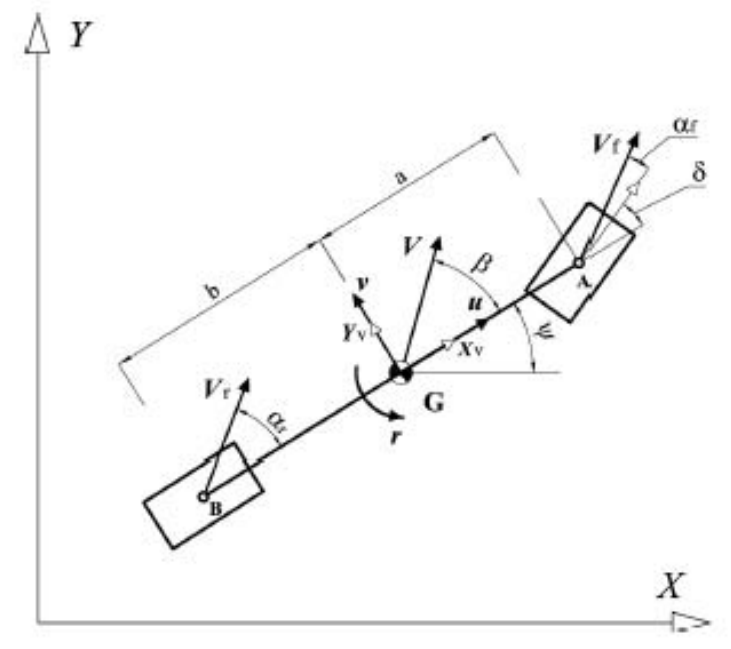

Figure 1: Lateral vehicle dynamics

refer to the sideslip angle $\beta$, defined as the angle between the vector velocity pertaining to the centre of mass and the longitudinal axis of the vehicle $X_{V}$ :

$$
\beta=\arctan \frac{v}{u}
$$

Note that in conventional models, the mass is usually treated as a fixed parameter that typically refers to the maximum load condition. In this study, $M$ is treated as a time-varying parameter. As a consequence, Eq. (1) expresses a non-linear relationship between $v, r$, and $M$.

The method proposed in this paper for online mass estimation is based on the use of a vertical gyroscope and a lateral accelerometer. Considering that both sensors are generally available onboard via the ESP system, this approach results particularly attractive in terms of cost-effectiveness, requiring only additional software efforts.

The gyro signal $r_{g}$ is generally subject to an offset error and needs to be mod- 
eled [21]. A good working approximation of gyro measurement is $r_{g}=r+b_{g}$. The relationship between accelerometer's measurement and the state variables is $a_{y}=\dot{v}+u r$.

The state evolution of the nonlinear system can be represented in compact matrix form as

$$
\dot{x}(t)=f(x(t), \delta(t))
$$

where $x$ is the state vector and $f($.$) is the state evolution function. x$ is given by

$$
x=\left[v, r, \dot{v}, \dot{r}, b_{g}, M\right]^{T}
$$

Similarly, if the measurement vector $z$ is introduced

$$
z=\left[\begin{array}{l}
r_{g} \\
a_{y}
\end{array}\right]
$$

a measurement equation can be drawn in compact matrix form as

$$
z(t)=h(x(t))
$$

82 In summary, Eq. (3) and Eq. (6) can serve as the basis for non-linear ${ }_{83}$ estimation methods. In the context of this problem, an extended Kalman

94 filter or EKF is found to be a good solution, as explained in the next section.

\section{as 4. Vehicle estimation}

${ }_{96}$ It is not always possible to directly measure all states describing the ve${ }_{87}$ hicle's dynamic behavior because of technical and/or economic reasons. In

${ }_{8}$ addition, some of the model parameters may be uncertain or change over so time. Nevertheless state/parameter estimation may be inferred by derivaso tion using other available sensors through the use of observers or virtual 
91 sensors. Observation means the extraction of information of a given variable

92 of interest that is not directly measurable by using only available sensor data.

93 The idea behind the proposed research is to implement a model of the real

s system in an onboard computer that runs in parallel with the system itself

95 providing estimation of a given set of states or variables of interest. One

96 challenge is that the system to be observed is usually excited by a stochastic

97 noise, due for example to imperfections in modeling the system. In addition,

98 sensor measurements may be biased and affected by their own stochastic

9 noise. Therefore, a stochastic closed-loop observer is necessary. One com-

100 mon solution is the Kalman filter whose scope in this study is extended to

101 mass estimation as well by incorporating $M$ in the state vector. The pro-

102 posed framework is of general value and may be easily modified to track

103 other time-varying parameters. In the proposed embodiment, explained in the block diagram of Figure 2, the Kalman filter-based observer runs in 105 parallel with the system. Driver commands, i.e. the steer angle and the 106 longitudinal speed $(\delta, u)$ and measurements of vehicles's response $\left(r_{g}, a_{y}\right)$ are 107 fed into the estimator that recursively estimates the states (i.e., $\beta$ and $r$ ) and 108 parameters (i.e., $M$ ) of the system online during normal vehicle manoeuver100 ing. Additionally, the estimator provides estimate of gyroscope's bias that 110 can be used for on-line sensor calibration.

\section{4.1. Model-based Extended Kalman observer}

The Kalman filter addresses the general problem of estimating the state of a discrete-time controlled process that is governed by a difference equation (i.e., Eq. (3)) with a measurement (i.e., Eq. (6)). The first step is to express 


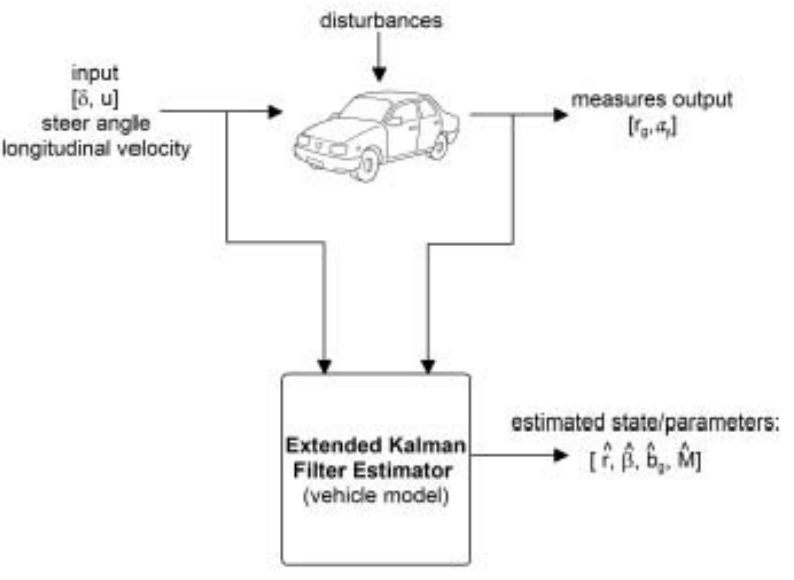

Figure 2: Block diagram of the proposed approach for state/parameter estimation using an EKF running in parallel

the non-linear model in a stochastic discrete-time state-space representation

$$
\begin{gathered}
x_{k}=f\left(x_{k-1}, \delta_{k}\right)+w_{k-1} \\
z_{k}=h\left(x_{k}\right)+v_{k}
\end{gathered}
$$

where $x_{k}=\left[v_{k}, r_{k}, \dot{v}_{k}, \dot{r}_{k}, b_{g, k}, M_{k}\right]^{T}$ is the state vector at time $k, \delta_{k}$ is the input vector at time $k$, and $z_{k}$ is the observation sampled at time $k$. If the system is discretised using the first-order Euler approximation with sampling 
time $\Delta t, f($.$) becomes$

$$
\begin{aligned}
& f_{1}: v_{k}=v_{k-1}+\dot{v}_{k-1} \Delta t \\
& f_{2}: r_{k}=r_{k-1}+\dot{r}_{k-1} \Delta t \\
& f_{3}: \dot{v}_{k}=-\left(\frac{C_{F}+C_{R}}{M_{k-1} u}\right) v_{k-1}-\left(\frac{C_{F} a-C_{R} b}{M_{k-1} u}+u\right) r_{k-1}+\frac{C_{F} \delta_{k}}{M_{k-1}} \\
& f_{4}: \dot{r}_{k}=-\left(\frac{C_{F} a+C_{R} b}{M_{k-1} a b u}\right) v_{k-1}-\left(\frac{C_{F} a^{2}+C_{R} b^{2}}{M_{k-1} a b u}\right) r_{k-1}+\frac{C_{F} a \delta_{k}}{M_{k-1} a b} \\
& f_{5}: b_{g, k}=b_{g, k-1} \\
& f_{6}: M_{k}=M_{k-1}
\end{aligned}
$$

whereas $h($.$) can be obtained as$

$$
\begin{aligned}
& h_{1}: r_{g, k}=r_{k} \\
& h_{2}: a_{y, k}=\dot{v}_{k}+u r_{k}
\end{aligned}
$$

The process disturbance and the measurement noise, $w_{k}$ and $v_{k}$, respectively, are assumed to be Gaussian, temporally independent of each other, and white, $Q$ and $R$ being the process and measurement noise covariance, respectively.

The Kalman filtering estimation operates through the prediction-correction cycle expressed by an a priori estimation:

$$
\begin{gathered}
\hat{x}_{k}^{-}=f\left(\hat{x}_{k-1}, \delta_{k}\right) \\
P_{k}^{-}=A_{k} P_{k-1} A_{k}^{T}+Q
\end{gathered}
$$

and a measurement update, which is only performed when the measurements are available, providing an a posteriori estimation:

$$
\begin{gathered}
K_{k}=P_{k}^{-} H_{k}^{T}\left(H_{k} P_{k}^{-} H_{k}^{T}+R\right)^{-1} \\
\hat{x}_{k}=\hat{x}_{k}^{-}+K_{k}\left(z_{k}-H_{k} \hat{x}_{k}^{-}\right) \\
P_{k}=\left(I-K_{k} H_{k}\right) P_{k}^{-}
\end{gathered}
$$


where $\hat{x}_{k}^{-}$is the predicted state vector, $P_{k}^{-}$is the variance matrix for $\hat{x}_{k}^{-}, K_{k}$ is the gain matrix, $\hat{x}_{k}$ is the updated state vector, and $P_{k}$ is the updated error covariance estimate. The prediction equations are responsible for projecting forward in time the current state and error covariance estimates to obtain the a priori estimate for the next time step. The correction equations are responsible for the feedback, i.e. for incorporating a new measurement into the $a$ priori estimate to obtain an improved a posteriori estimate.

In these equations, $A_{k}$ and $H_{k}$ are, respectively, the process and measurement Jacobian (matrix of partial derivatives of $f$ ( $h$, respectively) with respect to $x$ ) at step $k$ of the nonlinear equations around the estimated state

$$
\begin{gathered}
A_{k}=\left[\begin{array}{cccccc}
1 & 0 & \Delta t & 0 & 0 & 0 \\
0 & 1 & 0 & \Delta t & 0 & 0 \\
A_{31} & A_{32} & 0 & 0 & 0 & A_{36} \\
A_{41} & A_{42} & 0 & 0 & 0 & A_{46} \\
0 & 0 & 0 & 0 & 1 & 0 \\
0 & 0 & 0 & 0 & 0 & 1
\end{array}\right] \\
H_{k}=\left[\begin{array}{llllll}
0 & 1 & 0 & 0 & 1 & 0 \\
0 & u & 1 & 0 & 0 & 0
\end{array}\right]
\end{gathered}
$$

Please refer to the Appendix for the expression of the terms $A_{i, j}$ in $A_{k}$.

Finally, one should note that the measurement noise covariance $R$ is used to define the error of the sensor readings. Table 1 collects the sensor noise and bias used in this research that can be found on the sensor specification sheet or from observing static data from the sensor. 
Table 1: Sensor technical details

\begin{tabular}{lll}
\hline & Gyroscope & Accelerometer \\
\hline Sensor noise $(1 \sigma)$ & $1 \mathrm{deg} / \mathrm{s}$ & $0.5 \mathrm{~m} / \mathrm{s}^{2}$ \\
Output rate & $200 \mathrm{~Hz}$ & $200 \mathrm{~Hz}$ \\
Offset & $0.1 \mathrm{deg} / \mathrm{s}$ & - \\
\hline
\end{tabular}

117 4.2. Observability test for non-linear system

An important aspect of the state estimation problem is the observability. A system is said to be observable at a time step $k_{0}$ if, for a state $x\left(k_{0}\right)$ at that time, there is a finite $k_{1}>k_{0}$ such that knowledge of the output $z$ from $k_{0}$ to $k_{1}$ is sufficient to determine state $k_{0}$ [15].

The time derivative of measurement $z$ is:

$$
\frac{d z}{d t}=\frac{\partial h}{\partial x} \frac{d x}{d t}=\frac{\partial h}{\partial x} f(x)
$$

${ }_{118}$ Higher derivatives of $z$ can be written compactly by introducing the operator ${ }_{110} L_{f}$ (Lie derivative). $L_{f}[h]=\frac{\partial h}{\partial x} f(x)=$ time derivative of $h$ along the system trajectory $x$.

$$
\Rightarrow \frac{d^{2} z}{d t^{2}}=\frac{\partial}{\partial x}\left(\frac{\partial h}{\partial x} f(x)\right) f(x)=L_{f}\left[L_{f}[h]\right]=L_{f}^{2}[h]
$$

Therefore:

$$
\frac{d^{k} z}{d t^{k}}=L_{f}^{k}[h]
$$

A system with state vector $x$ of dimension $n$ is locally observable at $x_{0}$ if the observability matrix:

$$
O\left(x_{0}, \delta\right)=\left[d L_{f}^{0}[h]=d h, d L_{f}^{1}[h], \ldots, d L_{f}^{i}[h], . ., d L_{f}^{n-1}[h]\right]^{T}
$$


122 has row rank $n$ (i.e. $n$ linearly independent rows).

${ }_{123}$ In our case the observability matrix has dimension $n=6$. To calculate the 124 analytical expression of $O\left(x_{0}, \delta\right)$ the function $\operatorname{diff}($.$) of the Matlab Symbolic$ 125 tool is used. $O\left(x_{0}, \delta\right)$ resulted in full rank, therefore the system is locally observable.

\section{Results}

This section presents simulation results to show the effectiveness of the proposed approach for on-line mass estimation. In all simulations, the parameters of a typical passenger car are used (see Table 2). A common passing or double lane-change manoeuver is considered, where the driver quickly swerves into the passing lane to avoid a slower car or an obstacle and then immediately swerves back to avoid oncoming traffic. Passing can be expressed by a sine function for steering input

$$
\begin{aligned}
& \delta(t)=\delta_{0} \sin \omega t \\
& \omega=\frac{2 \pi L}{u}
\end{aligned}
$$

${ }_{128}$ where $L$ is the moving length during the lane change and $u$ is the forward velocity of the vehicle. For a speed of $80 \mathrm{~km} / \mathrm{h}, L=33 \mathrm{~m}$, and steering wheel angle comprised between -80 and 80 degrees (steering ratio $\tau=1 / 20$ ), the corresponding driver command and illustrative path are shown in Figure 3. The lateral behaviour of the vehicle is simulated by discretizing Eq. (3) 133 and Eq. (6) with process and sensor noise. The sensor measurements are corrupted with random noise of the standard deviation as claimed in the specification of the sensor (see Table 1). Additionally, a bias that is within the normal sensor's specification is added to the gyroscope. The measurements 


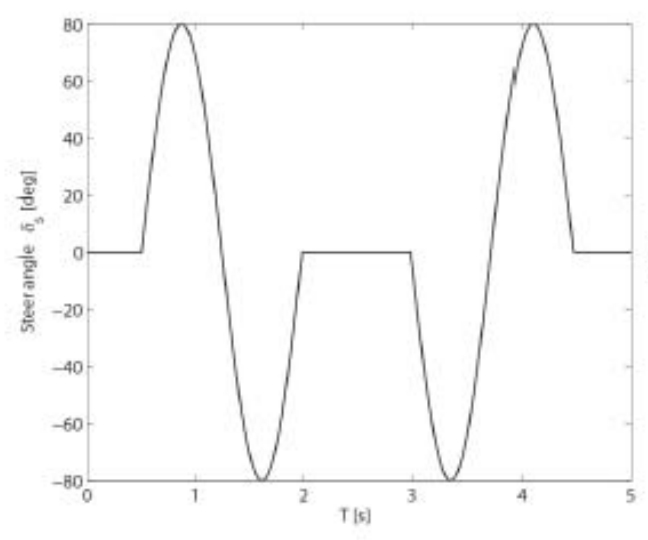

(a)

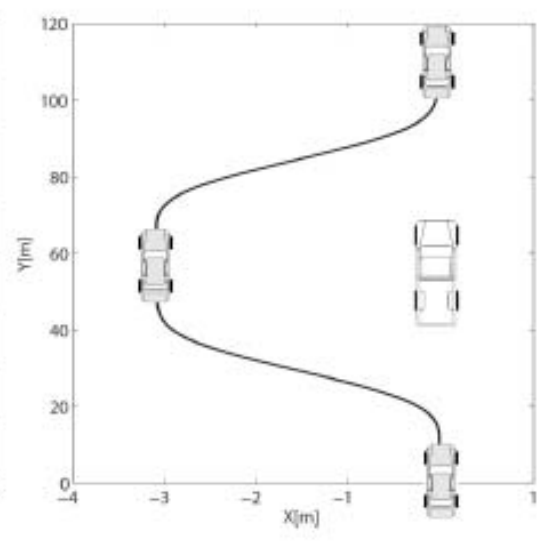

(b)

Figure 3: Double lane change: (a) steering wheel angle, (b) path followed by the vehicle.

are corrupted in order to provide realistic sensory input to the model-based observer. The correct process noise is given to the estimator.

\subsection{Parameter sensitivity}

First, a simulation is performed to show the effects of incorrect model parameters (i.e., vehicle load) on a conventional observer based on a "static" model with fixed parameters, i.e. without on-line mass estimation. The estimator model is given an incorrect value of mass increased of $20 \%$ with respect to the actual value ( $M=1400 \mathrm{~kg}$ ). Results are shown in Figure 4(a) in terms of simulated and estimated vehicle states $r$ and $\beta$ and they reveal how model parameter error leads to biased estimations of the states. One way to check the observer accuracy is to look at the residuals, i.e., the difference between the actual and the estimated measurements. The residuals for a correct observer should be white noise with zero mean. Conversely, in Figure 4(b) residuals for the rate of turn and lateral acceleration, as obtained from 
Table 2: Parameters of the passenger car used in the simulations, please refer to Figure 1 for more details

$$
\begin{array}{ll}
a & 1.108 \mathrm{~m} \\
b & 1.492 \mathrm{~m} \\
C_{F} & 117,240 \mathrm{~N} / \mathrm{rad} \\
C_{R} & 142,720 \mathrm{~N} / \mathrm{rad} \\
M & 1400-1683^{*} \mathrm{~kg} \\
I & M \cdot a \cdot b \\
\hline
\end{array}
$$

- The first and second value refer to the empty vehicle and maximum load, respectively

151 the observer with incorrect parameter, show a definite shape (or correlation).

152 The same manoeuver was repeated using the correct value of the vehicle mass 153 in the estimator. Results are collected in Figure 5(a), demonstrating that 154 estimation is very accurate with correct parameters even in the presence of noisy and biased sensor measurements. This is also confirmed when looking at the residuals of measurements that appear approximately as a zero mean 157 white noise (Figure 5(b)).

\subsection{Adaptive estimation}

Results presented in the previous section showed that the availability of an adaptive estimator for on-line mass estimation would be of great value to enhance the performance of on-board control systems by continuously updating the parameters of the vehicle model. A passing manoeuver simulation was performed using the adaptive observer proposed in this research. 

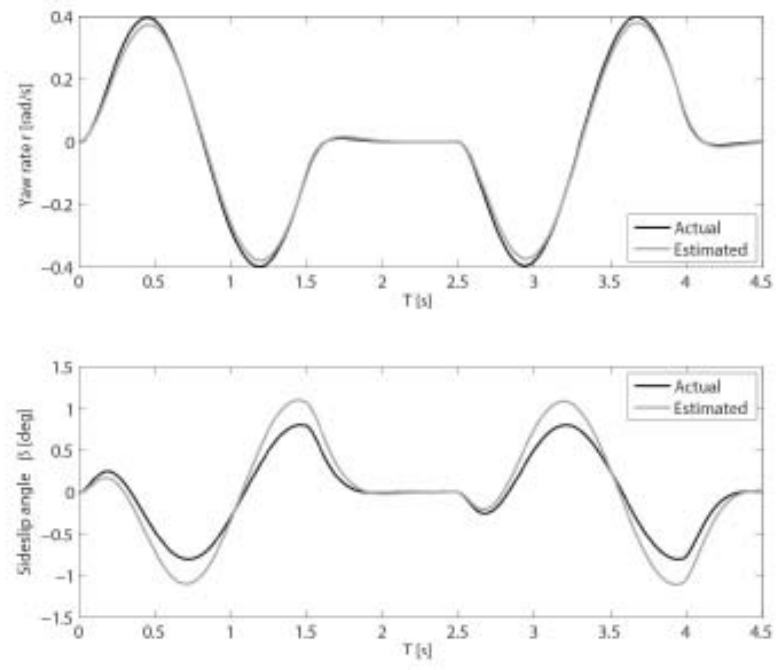

(a)
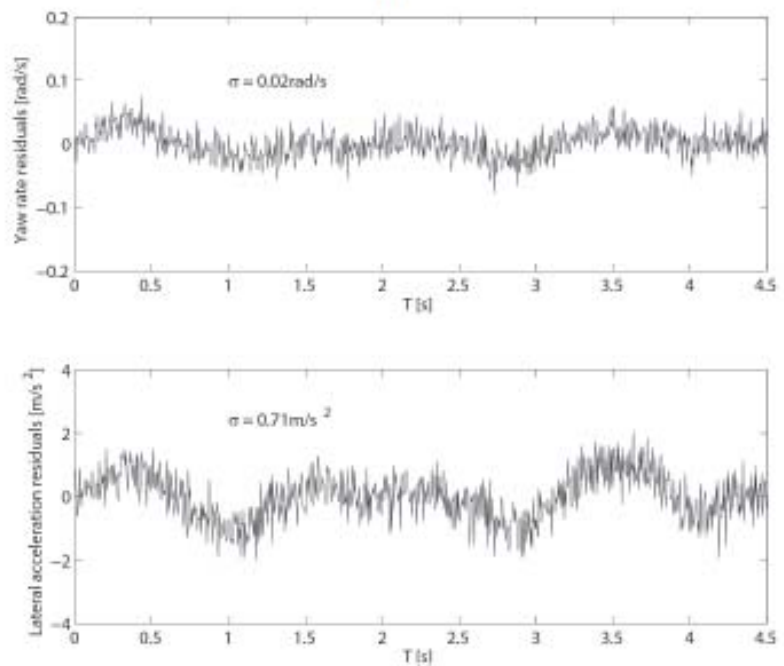

(b)

Figure 4: (a) State estimation and (b) residuals as obtained from a "static" estimator using incorrect parameters in the vehicle model for a double lane-change manoeuvre 

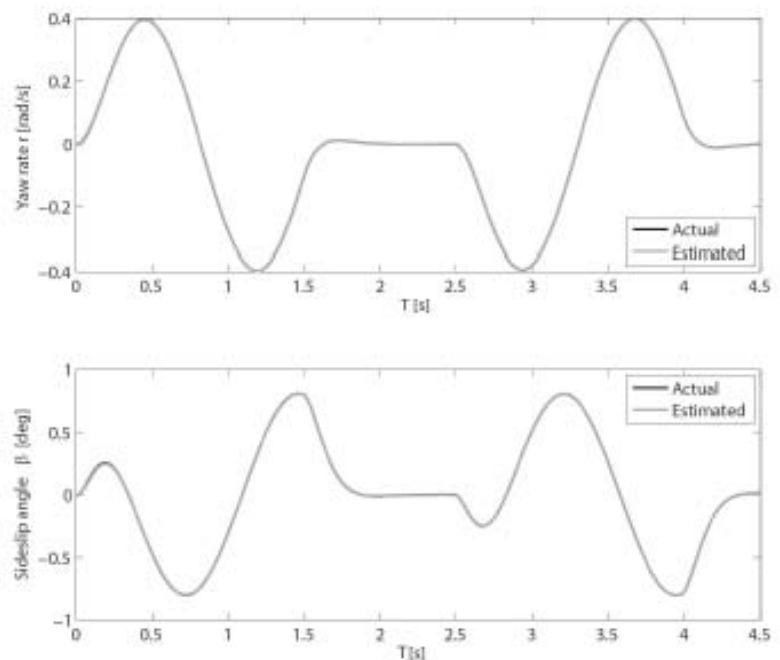

(a)
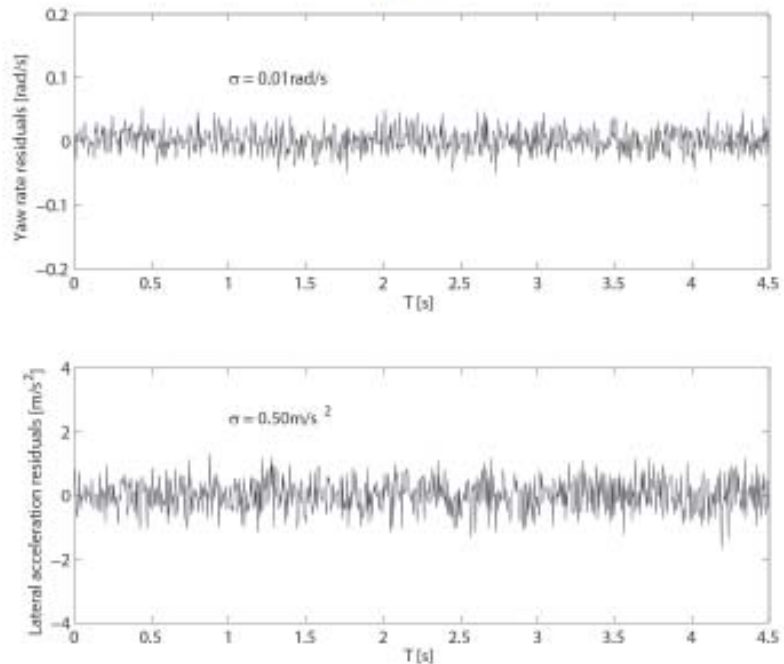

(b)

Figure 5: (a) State estimation and (b) residuals as obtained from a "static" estimator using exact parameters in the vehicle model for a double lane-change manoeuvre 
Initially, the observer is given an erroneous value of the vehicle mass corresponding to the maximum load condition, i.e., $M_{0}=1683 \mathrm{~kg}$. Estimation of states $r, \beta$ and $M$ as obtained from the observer is shown in Figure 6. In detail, the lower plot of Figure 6 shows the recursive mass estimation denoted by a solid grey line. The module corrects the parameter towards its actual value $M=1400 \mathrm{~kg}$ (denoted by a black dashed line), after an approximately 1 second adaptation window (the time required to reach $90 \%$ of the actual value is 0.39 seconds). As the system adjusts to the actual vehicle mass, the observer response becomes more accurate and the discrepancy of the state estimates $r$ and $\beta$ with respect to the actual values decreases, as shown in the upper and middle plot of Figure 6, respectively.

Uncertainty in the estimation, expressed as standard deviation $(3 \sigma)$, is also shown denoted by a grey shaded area along the corresponding curves. In detail, the variance of $r$ coincides with the term $P_{k}(2,2)$ of the error covariance matrix. The variance of $\beta, P_{k}^{\beta}$, can be obtained by applying the law of uncertainty propagation:

$$
P_{k}^{\beta}=J_{k} P_{k}^{v} J_{k}^{T}
$$

Where $J_{k}$ is the Jacobian matrix and $P_{k}^{v}$ is the covariance of the variables which $\beta$ depends on, i.e. the state $v$.

From Eq. (2), it results:

$$
\begin{gathered}
J_{k}=\left(\begin{array}{cc}
\frac{1}{u\left(1+\left(\frac{v}{u}\right)^{2}\right)} & 0
\end{array}\right) \\
P_{k}^{\beta}=J_{k}\left(\begin{array}{cc}
P_{k}(1,1) & 0 \\
0 & 0
\end{array}\right) J_{k}^{T}
\end{gathered}
$$

As seen in Figure 6, the estimation uncertainty tends to decrease as the accuracy in the mass estimation improves. 

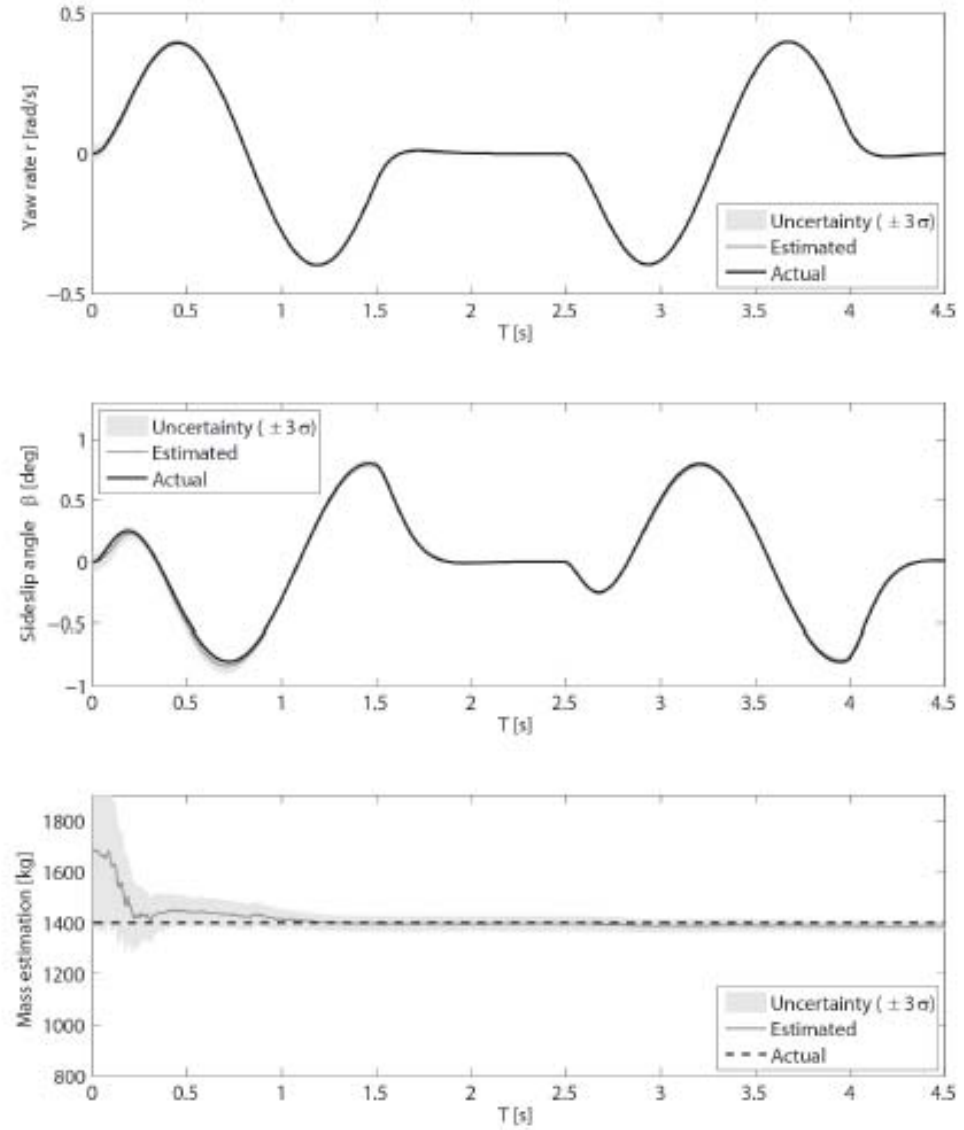

Figure 6: Results obtained from the proposed adaptive observer for a double lane-change manoeuvre. The accuracy in state estimation improves as the mass estimation tends to the actual value. 
161

162

163

164

180 its associated uncertainty.

181 The system responds well even in case of small changes in the vehicle load.

182 One additional advantage is that, the observer does not require any reset 183 procedure or learning stage that can be typically time consuming and difficult 184 to perform. 

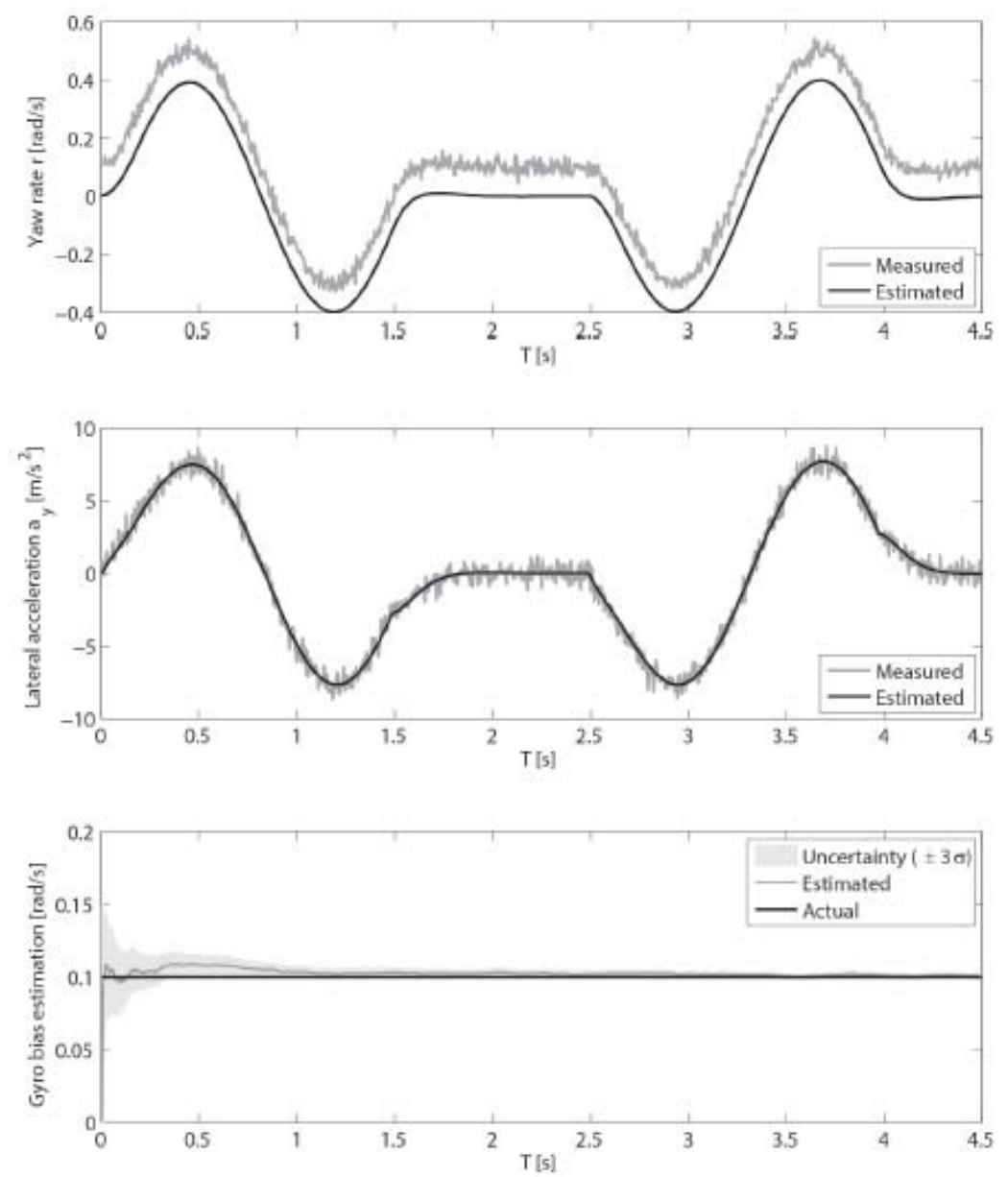

Figure 7: Results obtained from the proposed adaptive observer for a double lane-change manoeuvre. The noisy measurements are smoothed by the filter and the gyroscope bias is successfully updated on-line. 


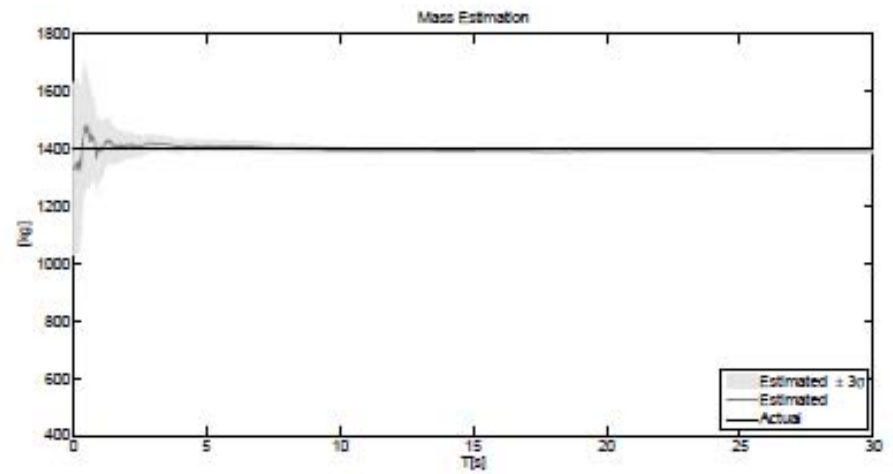

Figure 8: Minimum change of M...rifare figura con ingresso corretto

185

186

\section{Conclusions}

A model-based estimator for estimating vehicle's mass during normal driving and using only standard sensors was presented. The algorithm is based on a Extended Kalman filter to give robust and accurate estimates of the vehicle load and, at the same time, to allow abrupt changes to be tracked quickly. Results obtained from extensive simulation tests were presented to validate the proposed approach, using common manoeuvres (i.e., step-steer and double lane-change) and varying operating conditions (i.e., varying travel speed and steer angle). The sensitivity of the system was also studied. Especially, variations in the vehicle load can be detected quite accurately. The proposed approach could be useful to implement warning and safety systems and for accurate estimation of the vehicle states as possible input to onboard control systems.

a A possible limitation of this approach is that it may perform poorly under low excitation conditions. Before implementation, the system should be 


\section{Acknowledgments}

202 The financial support of the ER.A-NET ICT-AGRI2 through the grant S3-

203 CAV is gratefully acknowledged. This work has funded by the National Plan Project DPI2014-56364-C2-1-R.

205 Appendix

$$
\begin{aligned}
& A_{31}=-\frac{C_{F}+C_{R}}{M_{k-1}} \\
& A_{32}=-\left(\frac{C_{F u}-C_{R^{b}}}{M_{k-1}}+u\right) \\
& A_{11}=-\frac{C_{r a}^{a}+C_{n}^{b}}{M_{k-1} a b u} \\
& A_{42}=-\frac{C_{F} u^{2}+C_{R} b^{2}}{M_{k-1} a b e} \\
& A_{36}=\frac{C_{F}+C_{R}}{\hat{M}_{k-1}^{2}{ }^{k}} \hat{V}_{k-1}+\frac{C_{F} a-C_{n}^{b}}{M_{k-1}{ }^{u}} \hat{\gamma}_{k-1}-\frac{C_{F} \hat{d}_{k}}{M_{k-1}^{2}}
\end{aligned}
$$

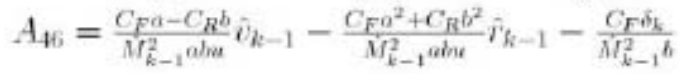

\section{References}

1) G. Reina, A. Messina, A. Gentile, Tyre pressure monitoring using a dynamical model-based estimator, Vehicle System Dynamics 53(4) (2015) 568586

[2] H. Bae, J. Gerdes, Parameter estlmation and command modification for longitudinal control of heavy vehicles, in: Proc. of International Symposium on Advanced Vehicle Control, 2000. 
[3] U. Kiencke, L. Nielson, Automotive Control Systems, Springer, New York, 2000.

[4] S. Yang, T. Liu, Y. Cheng, Automatic measurement of payload for heavy vehicles using strain gages, Measurement 41 (2008) 491-502.

[5] K. Nishitani, Electromagnetic-type load weigthing apparatus, United States Patent $5,243,146$.

[6] Continental-corporation, Intelligent tire sensors from continental will detect vehicle weight, On the WWW, uRL www. continental-corporation.com/www/pressportal_com_en/themes/press_releases/ (January 2013).

[7] M. Liubakka, D. Rhode, J. Winkelman, P. Kokotovic, Adaptive automotive speed control, IEEE Transactions on Automatic Control 38(7) (1993) $146-156$

[8] R. Rajamani, Vehicle Dynamics and Control, Springer, 2012.

[9] M. Druzhinina, A. Stefanopoulou, L. Moklegaard, Adaptive continuously variable compression braking control for heavy-duty vehicles, ASME Dynamic Systems, Measurement and Control 124(3) (2002) 406414.

[10] A. Vahidi, A. Stefanopoulou, H. Peng, Recursive least squares with forgetting for on-line estimation of vehicle mass and road grade: theory and experiments, Vehicle System Dynamics 43(1) (2005) 31-55. 
[11] P. Lingman, B. Schmidtbauer, Road slope and vehicle mass estimation using kalman filtering, in: Proceedings of the 17th IAVSD symposium, 2001.

[12] J. Holm, Vehicle mass and road grade estimation using kalman filter, Ph.D. thesis, Linkoping University, Sweden (2011).

[13] V. Winstead, I. V. Kolmanovsky, Estimation of road grade and vehicle mass via model predictive control, in: Proceedings of IEEE Conference on Control Applications, 2005.

[14] C. Kim, P. I. RO, Reduced-order modelling and parameter estimation for a quarter-car suspension system, Journal of Automobile Engineering 214 (2000) 851-864.

[15] M. Doumiati, A. Charara, A. Vicotrino, D. Lechner, Vehicle Dynamics Estimation using Kalamn Filtering, Wiley, 2013.

[16] A. E. Blanchard, C. Sandu, A polynomial chaos-based kalman filter approach for parameter estimation of mechanical systems, Journal of Dynamic Systems, Measurement and Control 132 (2010) 061404 (18 pp.).

[17] B. L. Pence, H. K. Fathy, J. L. Stein, Recursive estimation for reducedorder state-space models using polynomial chaos theory applied to vehicle mass estimation, IEEE Transactions on Control Systems Technology 22(1) (2014) 224-229.

[18] R. Tal, S. Elad, Method for determining weight of a vehicle in motion, United States Patent 034570. 
258 [19] E. Ritzen, Adaptive vehicle weight estimation, Ph.D. thesis, Linkoping 259 University, Sweden (1998).

260 [20] R. Jazar, Vehicle Dynamics, Springer-Verlag, New York, NY, USA, 2014.

261 [21] N. Persson, F. Gustafsson, M. Drevö, Indirect tire pressure monitoring ${ }_{262}$ using sensor fusion, in: SAE paper, 2002, pp. 1-6, paper number 2002$263 \quad 01-1250$. 